\title{
Effects of 20-H Rule and Shielding Vias on Electromagnetic Radiation From Printed Circuit Boards
}

\author{
Huabo Chen, Student Member, IEEE, and Jiayuan Fang, Senior Member, IEEE \\ Dept. of Electrical Engineering, University of California at Santa Cruz, Santa Cruz, CA 95064 \\ Tel: (831)459-4283 Fax: (831)459-4289 Email: hbchen@cse.ucsc.edu
}

Abstract: This paper investigates the effects of " $20-\mathrm{H}$ rule" and shielding vias on electromagnetic radiation from the printed circuits boards.

\section{Introduction}

As the operating frequency of electronic circuits continues to increase, today's package and printed circuit board designers face more radiation problem than ever before. The control of radiated emissions to make the package comply with radiation constraints is one of the most important aspects of the EMC study. Some "rules-of-thumb" are employed to help the designers to reduce the radiation problems. This paper presents an investigation on the effects of the $20-\mathrm{H}$ rule and the shielding vias on radiation from printed circuit boards.

\section{Effects of 20-H Rule}

The 20-H rule [1][3] states that the ground planes are extended beyond the power planes by about 20 times the distance between the planes. Let us consider the simple structure consisting of one power plane and one ground plane shown in Figure 1(a). The 20-H rule structure is shown in Figure 1(b).

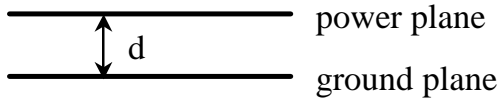

(a) two planes are of the same size

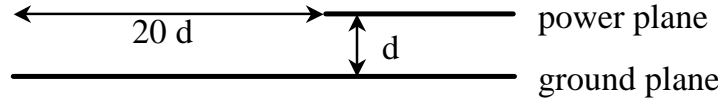

(b) $20-\mathrm{H}$ rule

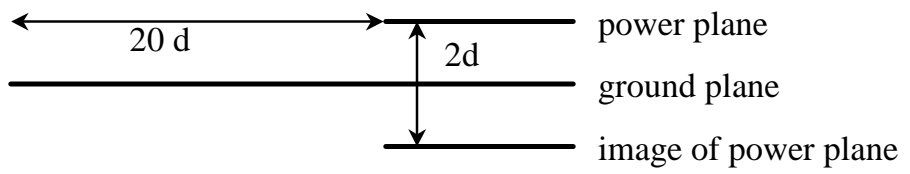

(c) image of the power plane in $20-\mathrm{H}$ rule structure

Figure 1

Since the radiation is closely related to reflection coefficient at the open end of the two planes, the reflection coefficient is then investigated. Assume the planes have zero thickness, the reflection coefficient at the open end of two parallel planes shown in Figure 1(a) can be found in [2]

$$
R=|R| e^{-j \theta},
$$

where

$$
\begin{gathered}
|R|=e^{-\pi q}, \\
\theta=2 q\left[1-C+\ln \frac{2}{q}-\left(\frac{\sin ^{-1} q}{q}-1\right)-\sum_{m=1}^{\infty} A_{2 m+1}\left(S_{2 m+1}-1\right) q^{2 m}\right],
\end{gathered}
$$

and

$$
q=\frac{d}{\lambda}
$$


where $C$ is Euler's constant, which is $0.577216, \lambda$ is the wavelength and $d$ is the plane separation. $A_{2 m+1}$ is the expansion coefficient of the function $\sin ^{-1} x$,

$$
\sin ^{-1} x=\sum_{m=0}^{\infty} A_{2 m+1} x^{2 m+1} .
$$

$S_{2 m+1}$ can be obtained by

$$
S_{2 m+1}=\sum_{n=1}^{\infty} \frac{1}{n^{2 m+1}} .
$$

The amplitude and phase of the reflection coefficient as functions of the plane separation $d$ are shown in Figure 2(a) and 2(b) respectively. It can be seen that the larger the separation between planes, the smaller the amplitude of the reflections coefficient and the larger the radiation. The reflection coefficient of the $20-\mathrm{H}$ rule structure can be estimated by that of the power plane and its image pair as shown in Figure 1(c). Because the separation between the power plane and its image plane is twice as large as that of the structure shown in Figure 1(a), the reflection coefficient of the $20-\mathrm{H}$ rule structure is smaller in the amplitude. Therefore, more radiation is expected to come out of the edges of the board implemented with 20-H rule.

The test structure for the numerical computation is shown in Figure 3. The size of the ground plane is $10 \mathrm{~cm}$ by $10 \mathrm{~cm}$ and the plane separation is $0.5 \mathrm{~mm}$. The relative dielectric constant is 4.0 . The power plane of the $20-\mathrm{H}$ rule structure is $1 \mathrm{~cm}$ smaller than the ground plane on each side. $3-\mathrm{D}$ FDTD method is used to compute the radiation. The radiation power is defined as the surface integration of the poynting vector

$$
P(t)=\int_{\text {Enclosed Surface }}(\vec{E} \times \vec{H}) \cdot d \vec{s}
$$

where $\vec{E}$ is the electric field and $\vec{H}$ is the magnetic field, on an enclosed surface of the board structure. The radiation is compared between the $20-\mathrm{H}$ rule structure and the normal structure where the ground plane and the power plane are both of $10 \mathrm{~cm} \times 10 \mathrm{~cm}$. Figure $4(\mathrm{a})$ shows the radiation on the top and the bottom surfaces. The radiation of the $20-\mathrm{H}$ rule structure is somewhat smaller on the bottom surface but increases significantly on the top surface. The total radiation on the enclosed surface is shown in Figure 4(b), from which one can see the radiation from the $20-\mathrm{H}$ rule board is much stronger than that from the board of the same size planes. This observation is consistent with the theoretical analysis presented above.

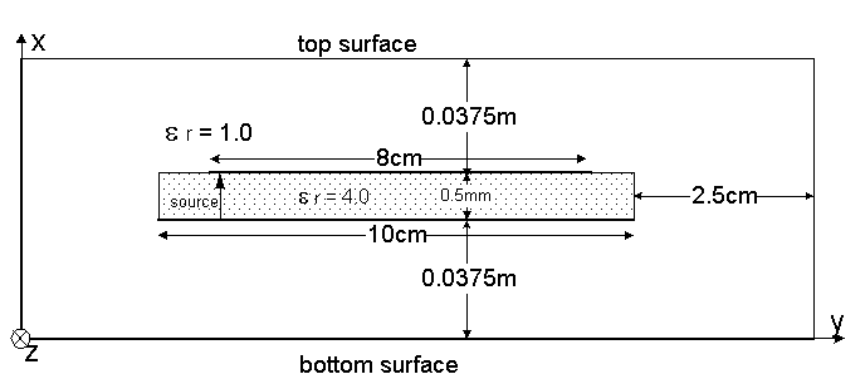

Figure 3 two-plane test structure

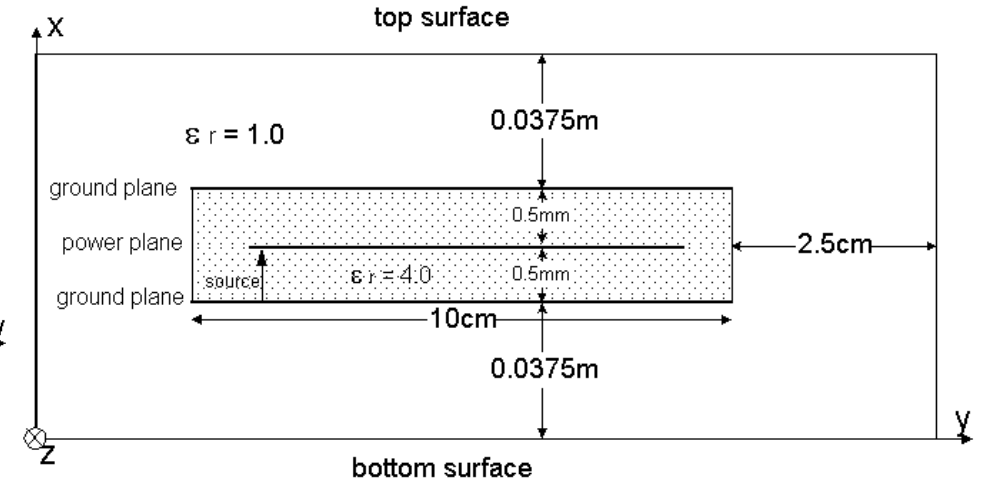

Figure 5 three-plane test structure

Consider the case where the $20-\mathrm{H}$ rule is applied to the three-plane structure shown in Figure 5. The top and bottom ground planes are of the same size. The power plane at the middle is shrinked inside by the "20-H rule". Radiation is examined on the enclosed surface. Figure 6(a) and (b) compare the radiation of the normal structure and the $20-\mathrm{H}$ rule structure on the top and the bottom surfaces. It is found that for the more than two plane structure, there is no significant change in radiation if the power plane in the middle is setback using $20-\mathrm{H}$ rule. 


\section{Effects of Shielding Vias}

The effects of shielding vias are also studied using FDTD method. Shielding vias are added to connect the two ground planes on four edges of the three-plane structure shown in Figure 5. The radiation from the board with different numbers of shielding vias is compared in Figure 7. It can be seen that a small number of shielding vias can cut the radiation significantly.

\section{Conclusion}

This paper investigates the effects of $20-\mathrm{H}$ rule and shielding vias on the radiation from the printed circuit board. For the two-plane structure, $20-\mathrm{H}$ rule yields much more radiation than the normal structure. For the multiple plane case, no significant change in radiation is found if the $20-\mathrm{H}$ rule is applied to the internal planes. Also the numerical result shows that the usage of shielding vias would cut down the radiation effectively.

\section{Reference}

[1] Mark I. Montrose, Printed Circuit Board Design Techniques for EMC Compliance, New York: IEEE Inc., 1996.

[2] I. A. Weinstein, The Theory of Diffraction and the Factorization Method (Generalized Wiener-Hopf Technique), Boulder: Golem Press, 1969.

[3] Dr. Zorica Pantic-Tanner \& Franz Gisin, Radiation from Edge Effects in Printed Circuit Boards (PCBs), presentation at the monthly chapter meeting of Santa Clara Valley Chapter of IEEE EMC Society, May, 2000.

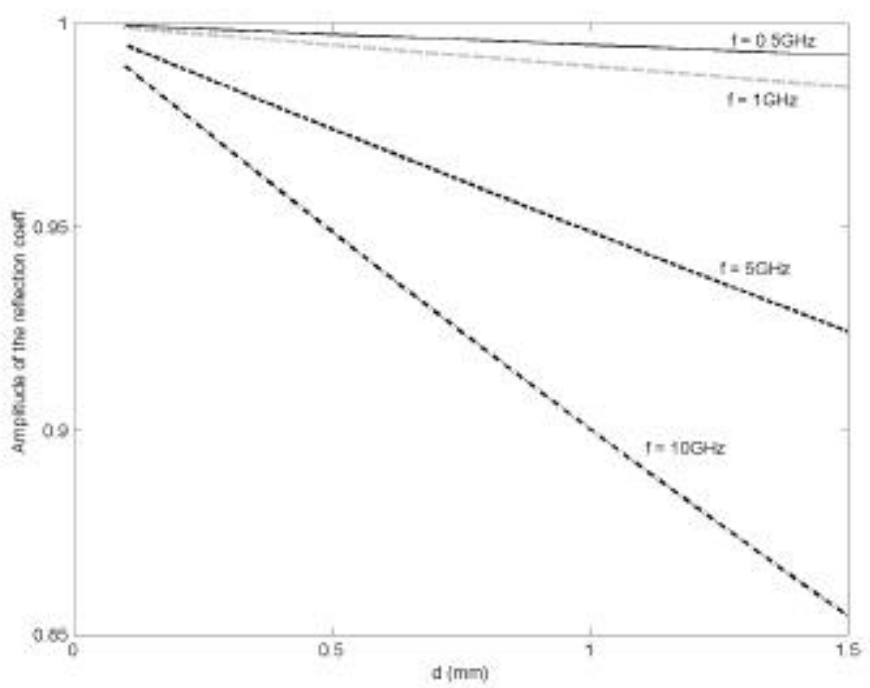

(a)

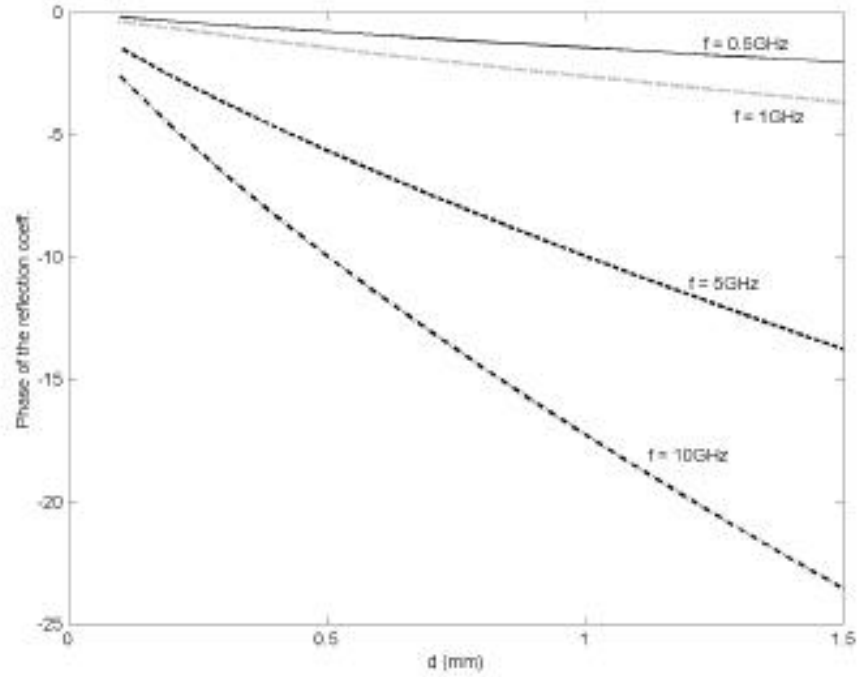

(b)

Figure 2. Reflection coefficient at the open end of two parallel planes (a) amplitude (b) phase 


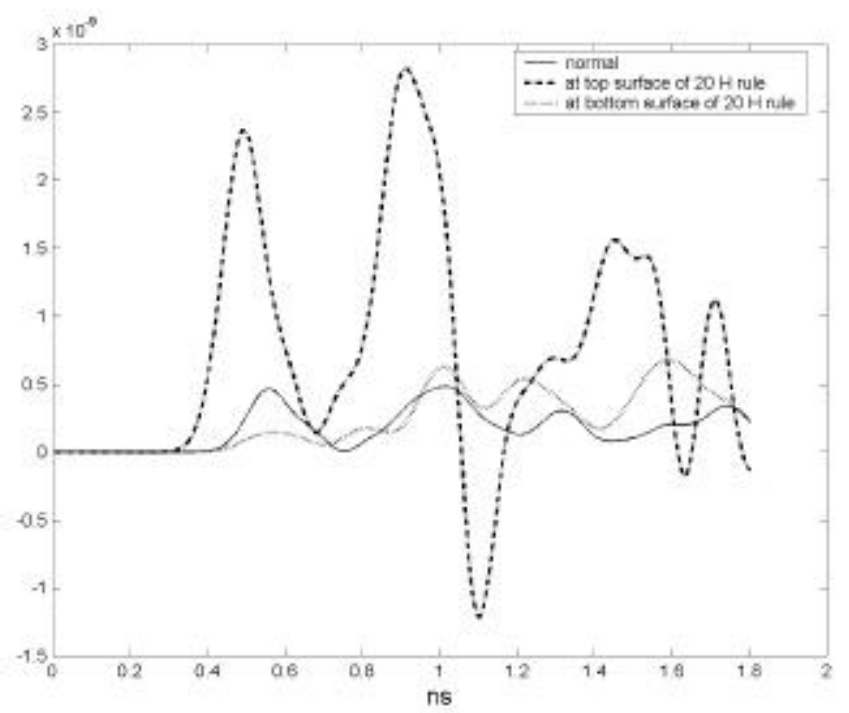

(a)

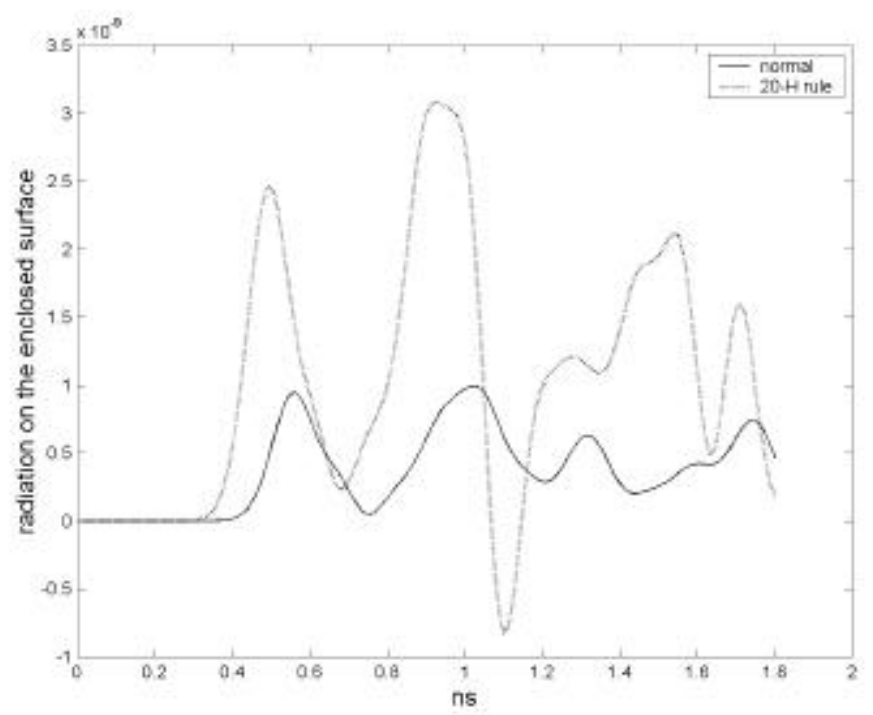

(b)

Figure 4. Comparison of the radiation of two-plane structure on (a) top and bottom surface (b) summation of all the surfaces

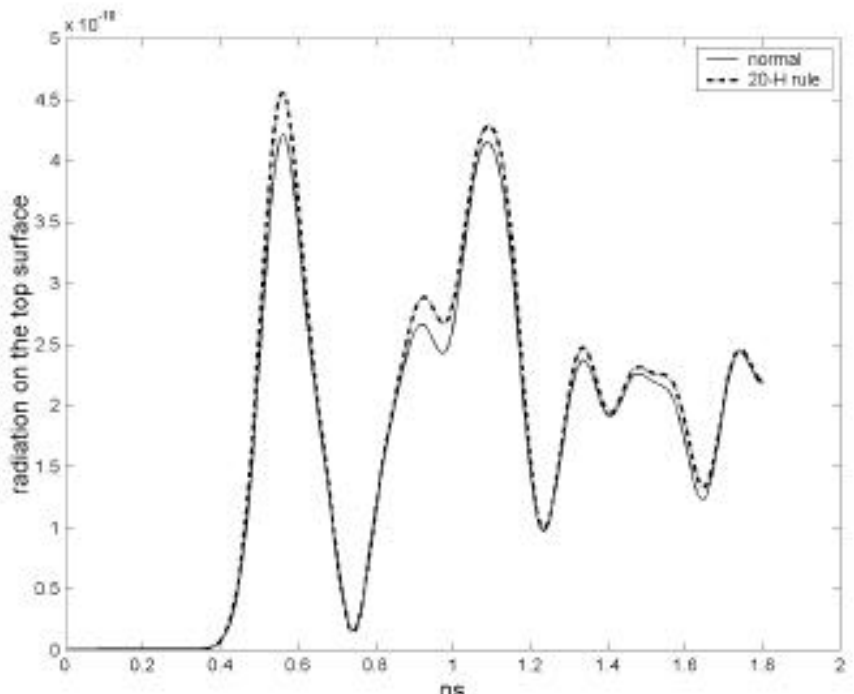

(a)

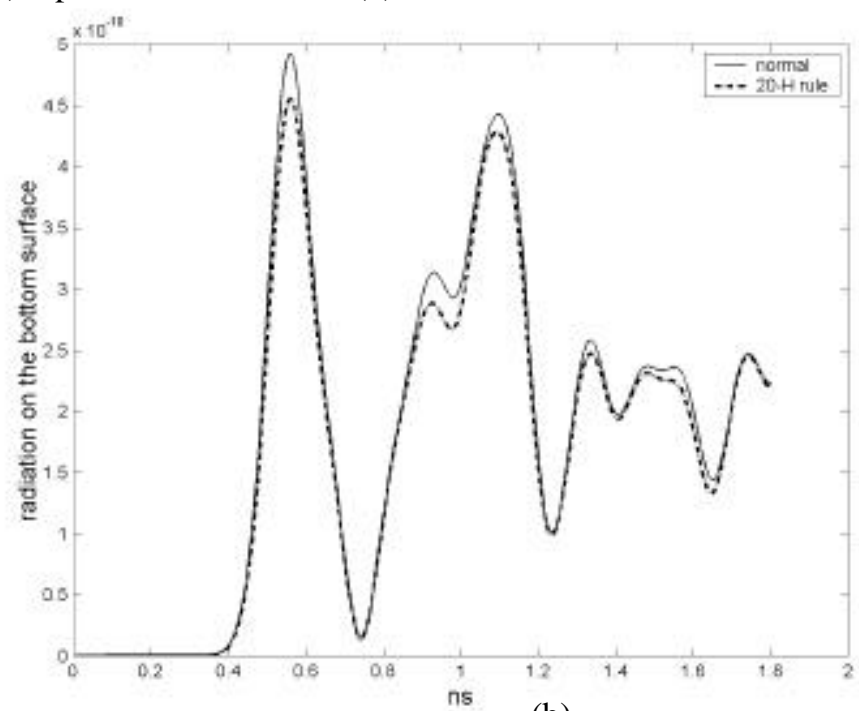

(b)

Figure 6. Comparison of the radiation of three-plane structure on (a) top surface (b) bottom surface

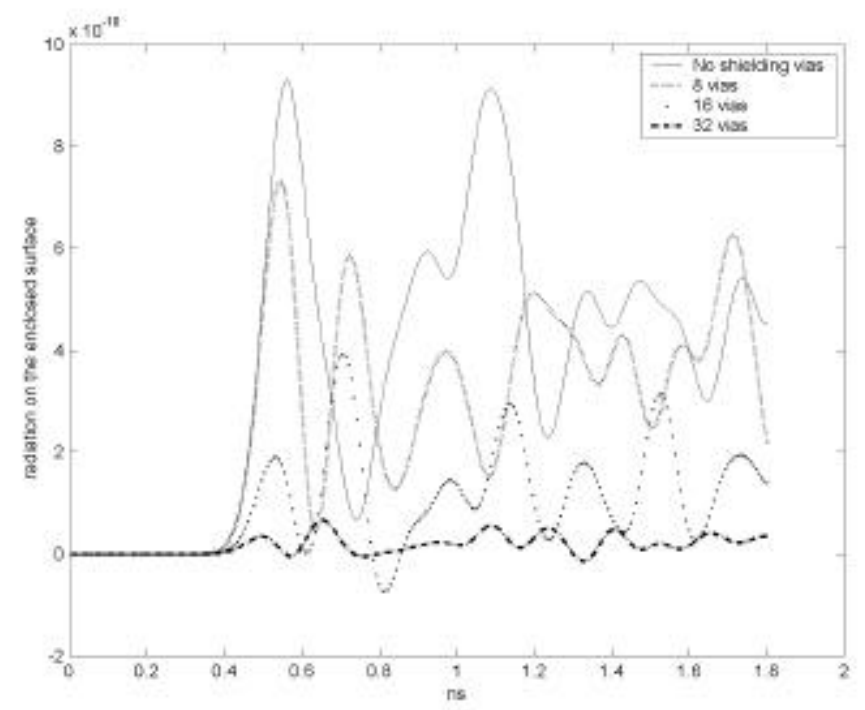

Figure 7. Radiation from the board with shielding vias 
